\title{
Contrast-Enhanced Ultrasound of Anaplastic Thyroid Cancer: A Case Report and Review of the Literature
}

\section{Introduction \\ $\nabla$}

Anaplastic thyroid cancer (ATC) is a very rare tumor that is associated with a high mortality rate (Nagaiah G et al. J Oncol $2011 ; 2011: 542358$ ). The majority of patients die within one year of diagnosis (Nagaiah G et al. 2011). The incidence, estimated to be around 1-2 cases per million/year, increases with age and peaks in the sixth and seventh decades of life. ATC represents approximately $2-5 \%$ of all cases of thyroid cancer and it is most prevalent in areas of iodine deficiency. In fact, many patients have a long clinical history of goiter and it has been hypothesized that prolonged TSH stimulation may be responsible for the transformation of a previously differentiated thyroid neoplasia into ATC. The histologic patterns of ATC include giant-cell, spindle-cell and squamoid-cell tumors. These subtypes frequently coexist and are not predictive of patient outcome. Histologically, approximately $20-30 \%$ of ATC cases show areas of necrosis and hemorrhage. Extensive coagulative necrosis with irregular borders is often seen. Infiltration of vascular walls is accompanied by obliteration of the vascular lumina (The International Agency for Research on Cancer, Pathology and Genetics of Tumours of Endocrine Organs 2004, 77-80).

We report a case of ATC studied with conventional B-mode ultrasound (US), color Doppler (CD) and contrast-enhanced ultrasonography (CEUS), and confirmed by fine needle-aspiration biopsy (FNAB). To the best of our knowledge according to the electronic database research (Pubmed), this is the first case reported in the literature describing the appearance of ATC at CEUS.

\section{Case Description \\ $\nabla$}

A 62-year-old woman was referred to our institution for an ultrasound of a palpable neck mass detected by physical examina-

\section{License terms}

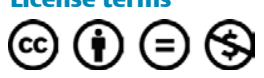

tion. The patient had normal levels of thyroid hormones and no history of previous surgery, radioactive iodine therapy, thyroid hormone suppression or family history of thyroid cancer. Conventional Bmode US was performed with a linear probe $(5-13 \mathrm{MHz}$ ) and an Alpha 10 ultrasound machine (Aloka, Tokyo, Japan). The examination showed a heterogeneously hypoechoic solitary nodule with a maximum diameter of $3 \mathrm{~cm}$ and irregular margins. There was no significant internal vascularity on color Doppler, but a few large peripheral vessels were noted in the surrounding thyroidal parenchyma (O Fig. 1). 3 ipsilateral round lymph nodes measuring $<1 \mathrm{~cm}$ were detected. CEUS was performed as a second level evaluation using both a convex probe $(2-6 \mathrm{MHz})$ and a linear probe $(5-13 \mathrm{MHz})$ compatible with contrast harmonic imaging. The convex probe was used to obtain a panoramic view, because of the large size of the nodule. The linear probe was used to investigate the microvascular flow pattern of the nodule. A bolus dose of $4.8 \mathrm{~mL}$ of a sulfur hexafluoride microbubble contrast agent (SonoVue; Bracco, Milan, Italy) was injected into an antecubital vein via a 20 -gauge cannula, followed by a $10 \mathrm{~mL}$ normal saline flush. CEUS was performed at a low mechanical index $(\mathrm{MI}<0.18)$, automatically defined by the software. The focus was placed deeper than the nodule plane to avoid rapid destruction of the microbubbles, and the tumor was continuously imaged for up to 2 min after injection. On CEUS, the nodule exhibited markedly reduced vascularity, except for a thin vessel at the periphery of the nodule. Time-intensity curves within selected regions of interest (ROI) and color maps were acquired using the software QONTRAST (Bracco, Milan, Italy). Values of contrast enhancement peak (peak) and time-to-peak (TTP) of the nodule and healthy thyroid tissue were calculated. Peak and TTP are reported as the index (peak index, TTP index) derived from the nodule/normal thyroid tissue ratio (Giusti et al. J Zhejiang Univ-Sci B 2013; 14: 195-206). According to the work of Giusti et al. (2013), a peak index less than 1 and a TTP index greater than 1 are indicative of malignancy. In this case of ATC, the average TTP index was 2 and the average peak index was 3.4. The frankly pathological TTP index is associated with curves that are shifted to the right compared to those commonly encountered in papillary carcinomas. Data relating to the behavior of anaplastic carcinoma in the literature are absent.

The grayscale US and CEUS findings raised suspicion of an underlying malignant lesion and FNAB was therefore performed. The final cytologic diagnosis was anaplastic thyroid cancer ( $\bullet$ Fig. 2 ). The patient underwent loco-regional radiotherapy with no response. She died 7 months after the diagnosis.

\section{Discussion}

$\nabla$

ATC has an aggressive behavior and, consequently, the latest American Joint Committee on Cancer Staging Manual classifies all ATCs as T4 and stage IV tumors, regardless of the actual overall tumor burden. In the majority of cases, ultrasound is the first diagnostic examination. Common US features of ATC nodules are a solid appearance, marked hypoechogenicity, irregular margins, internal calcifications, wider than tall shape and involvement of the cervical lymph nodes (The International Agency for Research on Cancer, Pathology and Genetics of Tumours of Endocrine Organs 2004, 77-80). However, US features of ATC overlap with those of other types of aggressive thyroid cancer and ultrasound-guided FNAB is mandatory for cytological confirmation, despite the great advances of thyroid nodule ultrasound techniques of US and US elastography (Cantisani $\mathrm{V}$ et al. Ultraschall Med. 2015 Apr; 36 (2): 162-167. Epub 2014 Jun 23, Cantisani et al. Eur Radiol. 2014 Jan; 24 (1): 143-150). Predominantly peripheral flow is suggestive of a benign nodule while central vascularity is suggestive of malignancy. There are no described features on color Doppler that are specific of anaplastic thyroid cancers. Other imaging techniques are generally used for staging. Computed tomography and PET with [18F] FDG can be employed to define the local extent of the disease and to detect lymph node metastases. Magnetic resonance is preferred over CT to detect invasion of vasculature, airway and bony structures.

While CEUS has been used extensively in the characterization of focal liver lesions because of its ability to differentiate be- 


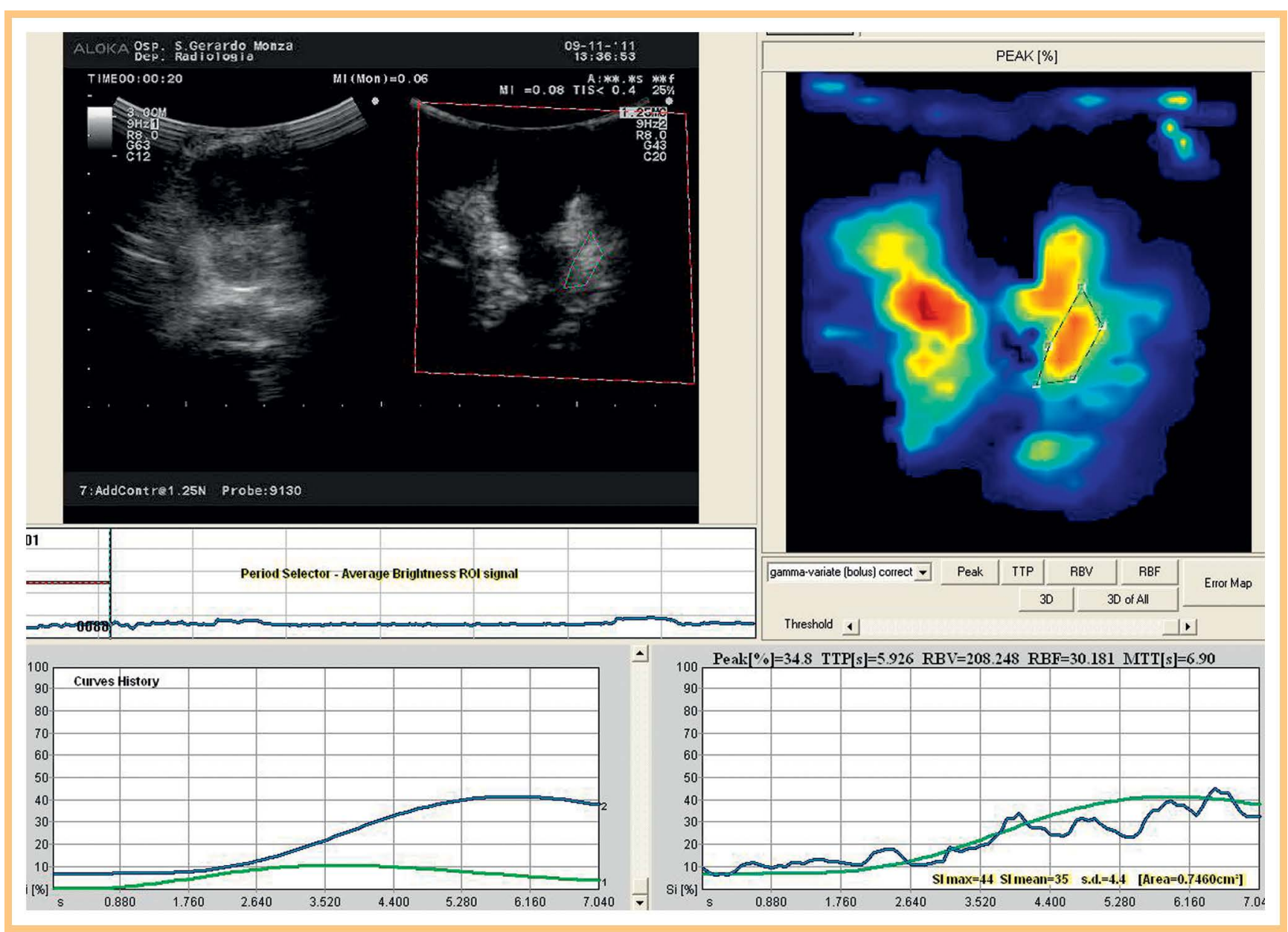

Fig. 1 a Grayscale (left) and contrast-enhanced (right) US paired images of an anaplastic thyroid cancer in a 62-year-old woman. On the contrast-enhanced image, the ROI, positioned on the normal thyroid parenchyma for quantitative analysis, is visible. The color maps based on the intensity of contrast b and the time-intensity curves $\mathbf{c}$ of the nodule (green) and normal parenchyma (blue) are presented. The curve of the ATC is characteristic of malignancy, opposite that of the healthy thyroid parenchyma. The color maps are used to calculate the peak index and TTP index of the tumor $\mathbf{d}$.

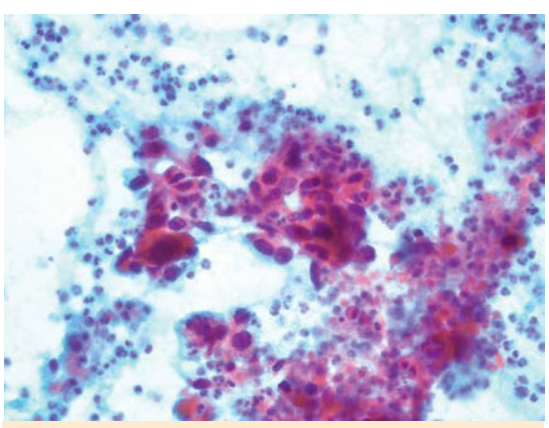

Fig. 2 Hematoxylin and eosin stained cytological smear from fine-needle aspiration biopsy of an anaplastic thyroid cancer in a 62-year-old woman. On a necrotic-inflammatory background (necrosis on the right and leucocytes and lymphocytes on the left), aggregates of large epithelial cells with well represented cytoplasm, marked atypia and nuclear pleomorphism are visible (arrows).

nign from malignant lesions based on different vascular patterns (Claudon $\mathrm{M}$ et al., Ultrasound Med Biol 2013; 39: 187-210), few studies have reported on the use of CEUS in the thyroid (U Nemec et al. Eur Radiol 2012; 22: 1357-1365, B Zhang et al. Thyroid 2010; 20: 51-57, Cantisani et al. Eur J Radiol. 2013 Nov; 82 (11): 1892-1898). Nevertheless, CEUS promises to be a useful adjunct to grayscale and color Doppler ultrasound in the assessment of thyroid nodules given its associated high sensitivity for differentiating benign from malignant thyroid nodules (U Nemec et al. 2012). In a recent study, malignant thyroid nodules exhibited 3 different enhancement patterns at CEUS evaluation (B Zhang et al. 2010): heterogeneous enhancement ( $88.2 \%$ of cases, with a reported specificity of $92.5 \%$ ), ring enhancement (5.9\%), and homogeneous enhancement (5.9\%). In contrast to those patterns of enhancement, CEUS of the anaplastic thyroid cancer reported here demonstrated almost a complete absence of vascularity, except for a thin peripheral vessel. The very poor vascularity of ATC on CEUS may be secondary to the typical infiltration of the vascular walls, obliteration of the vascular lumens and presence of necrosis. Even more recently, Yuan et al. (Yuan et al. J Cancer Res Ther. 2015 JanMar; 11 (1): 41-45) reported their experience in 78 patients with a solitary thyroid nodule (37 malignant, 41 benign) who underwent real-time CEUS. The patterns of their enhancement were assessed with regard to 6 aspects: Degree of enhancement, process of enhancement, homogeneity of enhancement, completeness of enhancement, boundary of the enhanced lesions, and shape of the enhanced lesions. They concluded that CEUS was accurate to discriminate benign from malignant lesions since they reported that the shape of most malignant lesions was irregular (94.59\%), the boundary was unclear $(86.49 \%)$ that $78 \%$ of the malignant lesions became inhomogeneously enhanced while $70 \%$ became incompletely enhanced. 
The CEUS findings of ATC described in this case report need confirmation by other cases to determine if this is a typical pattern that can be used to further characterize suspicious nodules on grayscale US as anaplastic thyroid cancer.

\section{Catchwords Phrases}

This is the first report of the CEUS appearance of an anaplastic thyroid cancer: the nodule was completely avascular.

This vascular pattern is different from the appearance of other thyroid cancers described in the literature as centrally vascularized.
Additional cases are needed to determine the specificity of the appearance of the anaplastic thyroid cancer reported here. Nevertheless, fine-needle aspiration remains the gold standard and is mandatory.

M. Proiti, Catania; A. Andreano, Milano;

S. Schiaffino, Genova; G. Turtulici, Genova;

P. Laeseke, Stanford; M. Meloni, Como

meloni.mariafranca@gmail.com 